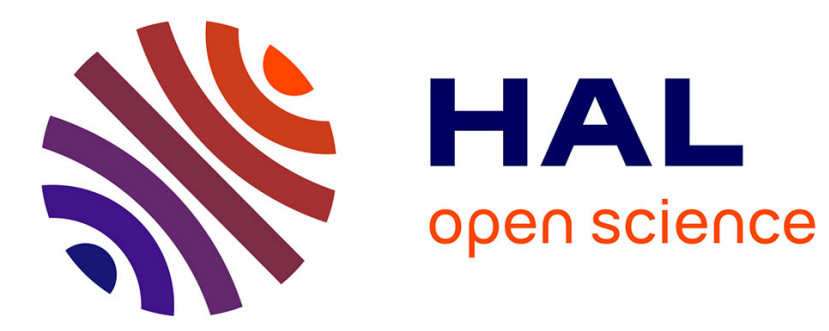

\title{
Mini-logique: logique rapide pour le traitement des informations en physique de haute énergie
}

\author{
S. Bréhin, J.C. Brisson, M. Lemoine, J.C. Raoul
}

\section{To cite this version:}

S. Bréhin, J.C. Brisson, M. Lemoine, J.C. Raoul. Mini-logique: logique rapide pour le traitement des informations en physique de haute énergie. Revue de Physique Appliquée, 1969, 4 (2), pp.157-158. 10.1051/rphysap:0196900402015700 . jpa-00243193

\section{HAL Id: jpa-00243193 https://hal.science/jpa-00243193}

Submitted on 1 Jan 1969

HAL is a multi-disciplinary open access archive for the deposit and dissemination of scientific research documents, whether they are published or not. The documents may come from teaching and research institutions in France or abroad, or from public or private research centers.
L'archive ouverte pluridisciplinaire HAL, est destinée au dépôt et à la diffusion de documents scientifiques de niveau recherche, publiés ou non, émanant des établissements d'enseignement et de recherche français ou étrangers, des laboratoires publics ou privés. 


\title{
MINI-LOGIQUE : LOGIQUE RAPIDE POUR LE TRAITEMENT DES INFORMATIONS EN PHYSIQUE DE HAUTE ÉNERGIE
}

\author{
S. BRÉHIN, J. C. BRISSON, M. LEMOINE et J. C. RAOUL, \\ Département de Physique des Particules Élémentaires, C.E.N., Saclay.
}

\begin{abstract}
Résumé. - L'utilisation de circuits intégrés rapides pour la construction de logiques de déclenchement de chambres à étincelles permet d'obtenir des temps de traitement des informations inférieurs à $100 \mathrm{~ns}$ avec des temps de résolution à l'entrée de $6 \mathrm{~ns}$. Le principe de la logique est détaillé et deux exemples sont présentés.
\end{abstract}

Abstract. - The use of fast integrated circuits to build spark chamber triggering logics allows one, to obtain a data processing time less than $100 \mathrm{~ns}$ with an input resolving time of 6 ns. The logical principle is detailed and two examples are given.

L'utilisation dans les expériences de haute énergie de chambres à étincelles associées à des hodoscopes groupant de 50 à 100 compteurs pose un problème pour la réalisation de la logique de déclenchement [1]. Le temps de mémoire des chambres (500 ns) exclut toute possibilité d'utilisation d'un calculateur classique. Cependant, l'utilisation des circuits intégrés rapides MECL de Motorola a permis de réaliser des logiques combinatoires inspirées des logiques de calculateur et dont le temps de traitement est inférieur à 100 ns. Ces minilogiques sont actuellement utilisées dans trois expé- riences : Hera (G.E.R.N., 1967/68), K $\mathrm{K}_{\mathrm{e} 4}$ (C.E.R.N., 1968) et $\gamma \mathrm{N}$ (Saturne, 1968).

La première fonction de cette logique est d'assurer un échantillonnage de l'information dans des circuits dits de filtrage (fig. 1). Ceux-ci sont constitués, sur chacune des voies, d'une porte ET en circuit intégré, dont le temps de résolution, qui était de $8 \mathrm{~ns}$ dans la première version, est maintenant de 6 ns et pourra sans doute être porté prochainement à 4 ns. Cette porte est suivie d'un discriminateur de mise en forme à diode tunnel et ligne à retard qui engendre une impulsion synchrone

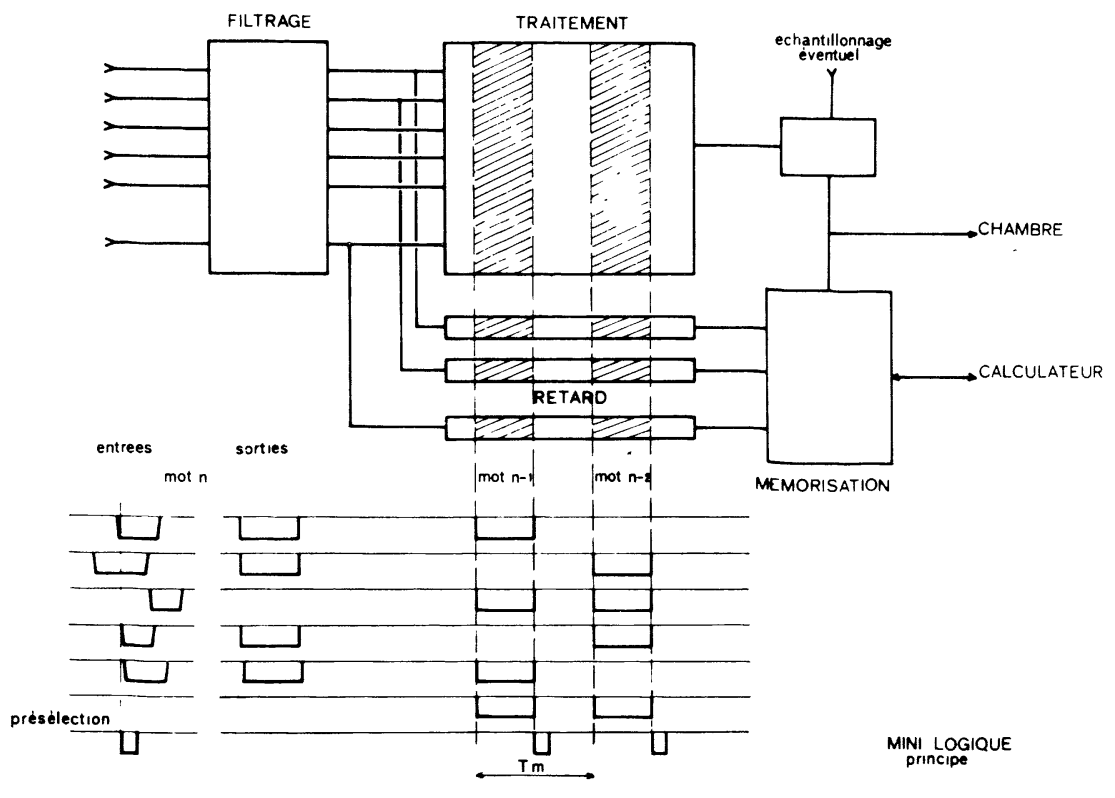

FIG. 1. - Principe de la logique. 
au signal d'échantillonnage et de durée parfaitement définie.

Ce circuit permet de s'affranchir dans la logique de traitement des ambiguïtés dues à la non-simultanéité des informations. En particulier, si certaines particules ont des temps-de-vol différents, le circuit restituera le synchronisme et ceci d'autant mieux que la résolution sera meilleure. D'autre part, ce circuit permet de réduire le taux de comptage à l'entrée de la logique de traitement. Chaque événement se présente à l'entrée de cette logique comme un mot possédant autant de digits qu'il y a de détecteurs. Le temps mort entre deux mots est égal à la durée du signal et le taux de comptage peut atteindre $50 \mathrm{MHz}$.

La seconde fonction est le traitement de ces informations en vue de la décision de déclenchement. Ce traitement est uniquement combinatoire et non séquentiel. De ce fait, le temps de traitement peut être supérieur au temps mort des circuits d'entrée et il peut y avoir simultanément plusieurs mots en cours de traitement dans la logique.

On peut sélectionner plusieurs classes d'événements. Comme cette logique est réalisée à partir de portes à entrées multiples, on peut envisager de faire effectuer des modifications de la logique par un calculateur connecté.

En général, on associe à l'information contenue dans la chambre à étincelles l'information sur les compteurs touchés dans l'événement. L'information mise en forme est stockée provisoirement dans une ligne à retard dont la longueur correspond au temps de traitement. A la sortie de la ligne, l'information est mémorisée ou non dans un bistable suivant le résultat du traitement. Cette sélection est faite avec un temps de résolution de $15 \mathrm{~ns}$. Ultérieurement, le calculateur viendra lire ces mémoires et les transférera soit sur une bande magnétique, soit sur la photographie de la chambre.
Nous allons maintenant voir deux exemples de logique de traitement :

1) Dans l'expérience Hera, on étudie, à l'aide d'une chambre à étincelles, la polarisation du proton de recul provenant de la diffusion élastique de pions sur les protons d'une cible polarisée. On utilise pour l'identification des événements 6 hodoscopes placés sur le trajet des particules. Deux critères sont à vérifier : les trois particules doivent être dans un même plan et les angles $\theta_{\mathrm{p}}$ du proton et $\theta_{\pi}$ du méson $\pi$ doivent être corrélés. Les angles $\theta_{\mathrm{p}}$ et $\theta_{\pi}$ sont des fonctions de la position des compteurs touchés dans les différents hodoscopes. A l'aide d'une matrice de circuits ET, on définit une fonction de deux variables. Un premier niveau de matrice permet de définir $\theta_{p}$ et $\theta_{\pi}$, une dernière matrice résout la fonction de corrélation.

2) Dans l'expérience $K_{\mathrm{e} 4}$, on s'intéresse à la désintégration d'un méson $\mathrm{K}^{+}$en 4 particules dont 3 sont chargées. L'événement est identifié par la détection de ces 3 particules dans l'hodoscope composé de 16 compteurs horizontaux et de 16 compteurs verticaux. Le traitement consiste à considérer chaque compteur d'hodoscope comme un bit (0 ou 1) et à additionner ces informations à travers une pyramide d'additionneurs binaires. On obtient finalement un mot de 5 digits dont la valeur représente le nombre de compteurs touchés.

Les modules sont très compacts, ce qui présente l'avantage de réduire au minimum les câbles d'interconnexion, la majorité de ces interconnexions se faisant à l'intérieur d'un même module par câblage imprimé. Le temps total de traitement est inférieur à $100 \mathrm{~ns}$. Le prix de revient est très faible comparé aux circuits classiques. Enfin, la fiabilité est excellente, ce qui est très important pour de gros ensembles.

\section{BIBLIOGRAPHIE}

[1] Raoul (J. C.), Coll. Intern. Électron. Nucl., Versailles, 1968, 3, 19-1. 